\title{
O adolescente e o uso de drogas
}

\author{
Ana Cecília Petta Roselli M arques ${ }^{a}$ e M arcelo S Cruz ${ }^{b}$
}

${ }^{a}$ Unidade de Dependência de Drogas do Departamento de Psicobiologia da Universidade Federal de São Paulo (UDED/Unifesp). ${ }^{\text {b} N u ́ c l e o ~ d e ~ E s t u d o s ~ e ~ P e s q u i-~}$ sas em Atenção ao Uso de Drogas da Universidade Estadual do Rio de Janeiro (NEPAD/UERJ)

\section{Introdução}

O uso de drogas é um fenômeno bastante antigo na história da humanidade e constitui um grave problema de saúde pública, com sérias consequiências pessoais e sociais no futuro dos jovens e de toda a sociedade.

A adolescência é um momento especial na vida do indivíduo. Nessa etapa, o jovem não aceita orientações, pois está testando a possibilidade de ser adulto, de ter poder e controle sobre si mesmo. É um momento de diferenciação em que "naturalmente" afasta-se da família e adere ao seu grupo de iguais. Se esse grupo estiver experimentalmente usando drogas, o pressiona a usar também. Ao entrar em contato com drogas nesse período de maior vulnerabilidade, expõe-se também a muitos riscos. $\mathrm{O}$ encontro do adolescente com a droga é um fenômeno muito mais frequiente do que se pensa e, por sua complexidade, difícil de ser abordado.

\section{Epidemiologia}

Os levantamentos epidemiológicos sobre o consumo de álcool e outras drogas entre os jovens no mundo e no Brasil mostram que é na passagem da infância para a adolescência que se inicia esse uso. Nos Estados Unidos, estima-se que cerca de três milhões de crianças e adolescentes fumem tabaco. $\mathrm{O}$ álcool é usado pelo menos uma vez por mês por mais de 50\% dos estudantes das últimas séries do que corresponde ao nosso ensino médio, sendo que $31 \%$ chega a se embriagar mensalmente. ${ }^{2}$ Dryfoos ${ }^{3}$ encontrou na população jovem americana (13 a 18 anos) as seguintes taxas de uso de tabaco, álcool e drogas: $12 \%$ de fumantes pesados (um maço ou mais ao dia); $15 \%$ de bebedores pesados (cinco ou mais doses por dia em três ou mais dias dos últimos 15); $5 \%$ fazem uso regular de maconha (20 ou mais dias no último mês); e 30\% fazem uso freqüente de cocaína (três ou mais vezes no último mês). ${ }^{4} \mathrm{O}$ uso de drogas varia de acordo com o sexo e, em meninos, esse uso aparece associado com mais frequiência à delinqüência..$^{5-7}$

No Brasil, o panorama mudou completamente nas últimas décadas. Até o início da década de 80, os estudos epidemiológicos não encontravam taxas de consumo alarmantes entre estudantes. ${ }^{8}$ No entanto, levantamentos realizados a partir de 1987 pelo Centro Brasileiro de Informações sobre as Drogas Psicotrópicas da Universidade Federal de São Paulo (CEBRID) têm documentado uma tendência ao crescimento do consumo. Esses levantamentos foram realizados entre estudantes de primeiro e segundo graus em dez capitais brasileiras e também em amostras de adolescentes internados e entre meninos de rua. Em 1997, o CEBRID mostrou que existe uma tendência ao aumento do consumo dos inalantes, da maconha, da cocaína e de crack em determinadas capitais. ${ }^{9-13}$ No entanto, o álcool e o tabaco continuam de longe a ocupar o primeiro lugar como as drogas mais utilizadas ao longo da vida e no momento atual (último mês) e com mais problemas associados, como por exemplo, os acidentes no trânsito e a violência. ${ }^{14}$

Estudo realizado em 1997 pelo Núcleo de Estudos e Pesquisas em Atenção ao Uso de Drogas da Universidade Estadual do Rio de Janeiro, avaliou 3.139 estudantes da quinta série do primeiro grau à terceira série do segundo grau de escolas públicas, possibilitando comparar as taxas de uso experimental ao longo da vida com as de uso habitual (últimos 30 dias). $\mathrm{O}$ estudo encontrou um consumo ao longo da vida e nos últimos 30 dias, respectivamente, de 77,7\% e 19,5\% para álcool; $34,9 \%$ e 4,6\% para tabaco; $9,2 \%$ e $2,8 \%$ para inalantes; $7,1 \%$ e $1,6 \%$ para tranqüilizantes; $6,3 \%$ e 2,0\% para maconha; e $1,9 \%$ e $0,6 \%$ para cocaína. ${ }^{15}$

Entre os fatores que desencadeiam o uso de drogas pelos adolescentes, os mais importantes são as emoções e os sentimentos associados a intenso sofrimento psíquico, como depressão, culpa, ansiedade exagerada e baixa auto-estima. ${ }^{16}$

\section{Psicofarmacologia}

Questões freqüentes relacionadas ao uso de álcool e drogas incluem os mecanismos de ação dessas substâncias, se o uso traz piores consequiências na população jovem e se existem drogas mais fortes ou piores que outras.

As pesquisas neurofisiológicas sugerem que as drogas psicotrópicas usadas de forma abusiva estimulam a ação dopaminérgica em vias mesolímbicas localizadas na área tegumentar ventral e no núcleo accumbens, o que teria papel determinante no estabelecimento de dependência. ${ }^{17}$ Além de agir sobre vias dopaminérgicas, cada substância age também em outros neurotransmissores, o que faz com que os vários tipos de drogas tenham efeitos diferentes. Assim, o álcool e outros depressores do sistema nervoso central, como os benzodiazepínicos, agem estimulando a neurotransmissão gabaérgica, provocando um efeito inicialmente desinibidor e posteriormente depressor. $\mathrm{O}$ álcool age também em receptores de glicina, glutamato (NMDA, AMPA e kainatos), acetilcolina (nicotínicos), proteína G, AMP cíclico e canais de cálcio. Os efeitos crônicos incluem uma ação na adenil ciclase e interferem na expressão genética e de fatores neurotrópicos. Não se sabe se esses efeitos teriam relação com o desenvolvimento de quadros como a síndrome alcoólica fetal e a neurotoxicidade no cérebro do adulto. ${ }^{18}$ 
Os prejuízos provocados pelas drogas podem ser agudos (durante a intoxicação ou "overdose") ou crônicos, produzindo alterações mais duradouras e até irreversíveis. O uso de drogas por adolescentes traz riscos adicionais aos que ocorrem com adultos em função de sua vulnerabilidade. Todas as substâncias psicoativas usadas de forma abusiva produzem aumento do risco de acidentes e da violência, por tornar mais frágeis os cuidados de autopreservação, já enfraquecidos entre adolescentes. Esses riscos ocorrem especialmente com o uso do álcool, a droga mais utilizada nessa faixa etária. $\mathrm{O}$ álcool pode causar intoxicações graves, além de hepatite e crises convulsivas.

O uso abusivo de benzodiazepínicos pode potencializar os efeitos do álcool e, em altas doses, provocar depressão respiratória. O uso crônico de benzodiazepínicos produz dependência e sua retirada abrupta pode provocar síndrome de abstinência. O risco do desenvolvimento desses quadros não deve ser negligenciado pelos médicos.

Os inalantes, como a cola de sapateiro, solventes de tinta, esmalte, benzina e lança-perfume incluem ampla gama de substâncias absorvidas pelos pulmões. As mortes durante intoxicações são raras, podendo acontecer por asfixia ou arritmias cardíacas. Várias síndromes neurológicas persistentes podem ocorrer com o uso crônico, principalmente neuropatia periférica, ototoxicicidade e encefalopatia. Também podem ocorrer lesões renais, pulmonares, hepáticas, cardíacas e no sistema hematopoiético.

A cocaína e as anfetaminas estimulam as ações dopaminérgica e noradrenérgica, podendo produzir, durante a intoxicação, crises convulsivas, isquemia cardíaca e cerebral, além de quadros maniformes e paranóides. O uso crônico induz a síndromes psiquiátricas semelhantes a depressão, ansiedade, pânico, mania, esquizofrenia e transtornos de personalidade. Também provoca piora do desempenho em tarefas que exigem a integridade de funções cognitivas, exaustão crônica e alterações funcionais de lobos frontais. O uso endovenoso está relacionado à transmissão de doenças como a síndrome de imunodeficiência adquirida (AIDS), e as hepatites B e C. Além das lesões já descritas que podem ser provocadas por outras formas de utilização da cocaína, o uso do crack pode provocar vários problemas pulmonares, como tosse, expectoração, pneumonia, hemoptise, bronquioespasmo e edema pulmonar. ${ }^{19}$ A cocaína e, principalmente, o "crack" são drogas que podem desenvolver dependência de forma rápida. Atividades ilícitas podem constituir o modo pelo qual crianças e adolescentes que não têm meios próprios adquirem as drogas. ${ }^{20}$

Segundo Hird et al, a maconha produziria a síndrome amotivacional, caracterizada por passividade, apatia, falta de objetivos, de ambição e de interesse na comunicação, podendo levar à queda do desempenho escolar, o que, por sua vez, pode aumentar a ansiedade, provocando aumento do uso. ${ }^{3}$

Entre os alucinógenos, o LSD age em vários neurotransmissores, mas sua ação sobre a serotonina parece ser a mais importante. Durante a intoxicação, quadros delirantes e alucinatórios aumentam o risco de acidentes, entre outros.

\section{Diagnóstico}

Outro aspecto muito importante desse tema é como realizar a identificação do jovem que usa drogas e tem problemas relacio- nados, o "adolescente de risco". O uso de drogas é um fenômeno multidimensional, que pode acontecer durante a adolescência, quando também podem surgir outros transtornos psicológicos, comportamentais e sociais. Entre as psicopatologias que mais incidem na puberdade (depressão maior, transtorno de déficit de atenção/hiperatividade e do comportamento disruptivo) detectam-se sinais e sintomas semelhantes àqueles também observados com o uso dessas substâncias, dificultando o diagnóstico diferencial. ${ }^{21,22}$ Assim, uma avaliação inicial cuidadosa do jovem que procura tratamento pode auxiliar o diagnóstico e melhorar o prognóstico, pois essa população não busca ajuda por conta própria, principalmente quando estão em dificuldades relacionadas ao uso de drogas. ${ }^{23-25}$ Eles pouco relacionam possíveis alterações de seu comportamento, pensamento e mesmo de seu funcionamento orgânico com o uso dessas substâncias, pois essas mudanças muitas vezes decorrem também da adolescência normal. Quando o fazem, minimizam ou negam as evidências e, dentro de uma postura ainda ambivalente, dizem que "isso não é nada" e que poderão resolver tudo sozinhos. Portanto, esse momento é muito especial e, dependendo da forma de abordar o problema pelos familiares, amigos ou mesmo pelo profissional, a resistência pode aumentar e a chance de intervir diminuir. Portanto, o primeiro passo da intervenção com um jovem é adequar esse contato, por meio de uma entrevista afetiva, ativa, objetiva e clara, buscando a cooperação do paciente e reforçando o sigilo das informações. Deve-se propiciar uma anamnese livre, na qual o jovem responda a duas questões básicas: por que ele veio para a consulta e o que ele pensa que está errado com ele. O profissional deve conduzir esse contato tentando vencer a resistência do jovem e obtendo as informações necessárias para um diagnóstico mais preciso. A confidencialidade e a importância da percepção por parte do adolescente de que tem um papel a assumir no processo de mudança que ali se inicia são amplamente debatidos e garantidos. Esses cuidados são imprescindíveis para desenvolver um bom rapport, o objetivo principal dessa primeira entrevista.

São objetivos dessa avaliação: estabelecer o vínculo; investigar sobre a saúde física e mental; sobre o comportamento e o relacionamento social e familiar; o ajustamento escolar ou profissional; sobre seu lazer; e, finalmente, sobre o uso de drogas e os problemas a ele associados, estabelecendo uma história sobre o uso de drogas na vida. Após essa avaliação global do adolescente, por meio da investigação das diversas áreas de sua vida, realiza-se o exame físico e solicitam-se exames laboratoriais, se necessário. O jovem deve receber todos os resultados dessa investigação. A seguir, define-se a gravidade do uso de drogas e suas conseqüências, desenvolvendo um plano de intervenção subseqüente, com metas e critérios de sucesso esperados com o tratamento. Se não for possível aplicar tal estratégia, é melhor encaminhar o jovem para um serviço especializado.

Sabe-se da importância do sistema familiar nas intervenções para prevenção e tratamento da dependência de álcool e outras drogas. Para a maioria dos jovens, o suporte socioeconômico vem dos pais e, para eles, os serviços de tratamento devem um esclarecimento legal sobre alguns problemas. Garantindo ao jovem o sigilo das informações pessoais, os pais devem saber 
compulsoriamente sobre risco de suicídio, síndrome de abstinência grave, intoxicação grave e abuso sexual. Muitas famílias também devem ser inseridas no tratamento.

Em função da complexidade da questão, é muito importante que se utilize questionários, inventários e escalas desenvolvidos para o jovem, com o objetivo de fundamentar o diagnóstico e o encaminhamento do caso. ${ }^{26,27}$ Para o diagnóstico, recomenda-se a Classificação de Transtornos Mentais e de Comportamento da Organização Mundial da Saúde (CID-10, WHO, 1992). No capítulo sobre transtornos mentais e de comportamento decorrentes do uso de substâncias psicoativas (F10 a 19), encontram-se os critérios diagnósticos para vários estados, sendo os mais importantes: intoxicação aguda, uso nocivo, síndrome de dependência, estado de abstinência, entre outros. Um diagnóstico de síndrome de dependência usualmente só deve ser feito se três ou mais dos seguintes requisitos estiveram presentes durante o último ano: a) um forte desejo ou senso de compulsão para consumir a substância; b) dificuldades em controlar o comportamento de consumir a substância em termos de seu início, término ou níveis de consumo; c) um estado de abstinência fisiológico quando o uso da substância cessou ou foi reduzido, como evidenciado por: a síndrome de abstinência característica para a substância ou o uso a mesma substância (ou de uma substância intimamente relacionada) com a intenção de aliviar ou evitar sintomas de abstinência; d) evidência de tolerância, de tal forma que doses crescentes da substância psicoativa são requeridas para alcançar efeitos originalmente produzidos por doses mais baixas (exemplos claros disso são encontrados em indivíduos dependentes de álcool e de opiáceos, que podem tomar doses diárias suficientes para matar ou incapacitar usuários não tolerantes); e) abandono progressivo de prazeres ou interesses alternativos em favor do uso da substância psicoativa, aumento da quantidade de tempo necessária para obter ou tomar a substância ou para se recuperar de seus efeitos; e f) persistência do uso da substância, a despeito da evidência clara de conseqüências manifestamente nocivas. Deve-se fazer esforços para determinar se o usuário estava realmente (ou se poderia esperar que estivesse) consciente da natureza e extensão do dano.

\section{Tratamento}

Como tratar o adolescente com problemas relacionados ao uso de álcool ou outras drogas? Os estudos de metanálise sobre a efetividade dos diversos tratamentos psicoterápicos para adolescentes conseguiram reunir em torno de 400 tipos diferentes de terapias utilizadas para adolescentes. ${ }^{28}$ Além dessa diversidade de intervenções, a escolha do tratamento dependeu de fatores extrínsecos, isto é, da disponibilidade do tratamento mais adequado para o jovem (próximo ao local de sua residência e compatível com sua condição socioeconômica e com seu sistema familiar), como também de fatores intrínsecos, como a motivação do jovem e a gravidade de seu diagnóstico como um todo. O tratamento do adolescente deve levar em consideração também, o tipo da droga utilizada e a freqüência do consumo. ${ }^{29}$

Até 1974, os adolescentes dependentes de álcool ou outras drogas recebiam tratamentos desenvolvidos originalmente para adultos. Wheeler e Malmquist ${ }^{30}$ propuseram o primeiro tratamento para jovens dependentes de álcool em regime de internação (28 dias), utilizando o modelo Minnesota, uma intervenção em grupo com o programa dos 12 passos dos Alcoóli$\cos$ Anônimos. ${ }^{30}$ Estes autores levaram em consideração algumas diferenças entre o adolescente e o adulto, aplicando uma técnica essencialmente comportamental e diretiva.

Cerca de $80 \%$ dos jovens com problemas associados ao uso de drogas são tratados em ambulatórios por meio de abordagens individual, grupal, familiar ou uma combinação dessas, aplicando-se modelos teóricos variados. ${ }^{31} \mathrm{O}$ tratamento pode ser feito em regime de internação parcial (hospital-dia) e em regime de internação integral, utilizando-se a psicanálise, a terapia comportamental, a cognitivo-comportamental, a interacional e a sistêmica, entre outras. ${ }^{32,33}$

Nas abordagens psicodinâmicas são privilegiadas formas de tratamento que promovam o desenvolvimento de modos mais satisfatórios de relação consigo mesmo e com os outros. Dessa forma, o recurso ao uso da substância deixa de ter a função anteriormente utilizada, ou seja, a resolução temporária de motivações inconscientes. Outras formas de tratamento se associam à psicoterapia, que pode tornar possível o encontro do indivíduo com aspectos seus anteriormente inacessíveis ao seu consciente. Tal encontro possibilita que o indivíduo ultrapasse impasses existenciais, vá além das repetições inconscientes de comportamentos que impedem o desenvolvimento de sua maturidade e autonomia e permite que ele expanda o seu repertório de recursos para enfrentar as vicissitudes do dia-a-dia. ${ }^{34}$

Já o modelo mais utilizado e recomendado entre os norteamericanos e os ingleses é o da terapia cognitivo-comportamental. ${ }^{35,36}$ A Teoria do Aprendizado Social de Bandura ${ }^{37}$ é a base teórica dessa intervenção, sendo o uso de drogas considerado um comportamento aprendido, desencadeado e mantido por eventos e emoções específicos e, portanto, possível de ser modificado. A família é considerada parte dessa disfunção e deve ser abordada.

Qualquer que seja o modelo teórico, o tratamento deve estar estruturado em três níveis: o desenvolvimento global do adolescente; a modificação do comportamento de uso de álcool ou drogas e a resolução dos problemas associados, além do reajuste familiar, social e ambiental.

O tratamento do dependente de substâncias psicoativas é bastante complexo e os estudos sobre a efetividade dos tratamentos para essa população adolescente devem ser replicados, pois os resultados ainda são pouco animadores. ${ }^{38}$ Para a população adulta, a literatura mostra que tratar é melhor que não tratar, mas não existe nenhum tratamento mais efetivo até o momento. ${ }^{39}$ A recaída, o desejo pela droga (a "fissura"), o pouco envolvimento nas tarefas escolares ou no trabalho, o lazer insatisfatório, a polidependência, o início de uso do álcool muito cedo na vida, as alterações de comportamento e o envolvimento criminal são fatores que contribuem para tornar o tratamento menos efetivo. ${ }^{40,41} \mathrm{~A}$ abstinência e o redimensionamento do funcionamento escolar, familiar e social são recomendados para aumentar a efetividade das intervenções. ${ }^{42}$ 


\section{Conclusão}

A identificação do adolescente de risco em função do uso de álcool ou drogas e a definição do melhor tratamento ainda são assuntos bastante complexos e alvo de muitas discussões. Algumas características do adolescente de risco podem auxiliar os trabalhos preventivos e de triagem para minimizar esse problema. Segundo Newcomb (1995), os fatores de risco para o uso de drogas incluem aspectos culturais, interpessoais, psicológicos e biológicos. São eles: a disponibilidade das substâncias, as leis, as normas sociais, as privações econômicas extremas; o uso de drogas ou atitudes positivas frente às drogas pela família, conflitos familiares graves; comportamento problemá- tico (agressivo, alienado, rebelde), baixo aproveitamento escolar, alienação, atitude favorável em relação ao uso, início precoce do uso; susceptibilidade herdada ao uso e vulnerabilidade ao efeito de drogas.

Pesquisas etnográficas e epidemiológicas utilizando uma metodologia rigorosa podem fundamentar projetos e prevenção em todos os níveis, fornecendo dados e elucidando muitas questões, pois o custo pessoal e social com a dependência nos países desenvolvidos tem sido muito maior que o gasto com a prevenção. No Brasil, mesmo sem tradição nessa área, é preciso priorizar políticas preventivas, gerando projetos mais ajustados à realidade brasileira, pois prevenir ainda é melhor que remediar!

\section{Referências}

1. Institute of Medicine. Treating drug problems: a study of the evolution, effectiveness, and financing of public and private drug treatment systems. Report prepared by the Institute of Medicine, Committee for the Substance Abuse Coverage Study, Division of Health Care Services. Washington (DC): National Academy Press; 1990.

2. Hird S, Khuri E, Dusenbury L, Millman RB. Adolescents. In: Lowinson JH, Ruiz P, Millman RB, Langrod JG. Substance abuse. A comprehensive textbook. $3^{\text {rd }}$ ed. Baltimore: Williams \& Willkins; 1997. p. 683-92.

3. Dryfoos JG. Adolescents at risk: prevalence and prevention. New York: Oxford University Press; 1990.

4. Weinberg NZ, Rahdert E, Colliver JD, Glanz MD. Adolescent substance abuse: a review of the past 10 years. J Am Acad Child Adolesc Psychiatry 1998;37:252-61.

5. Caron C, Rutter M. Comorbidity in child psychopathology: concepts, issues, and research strategies. J Child Psychopathol Psychiatry 1991;32:1063-80.

6. Offord D, Boyle MH, Racine YA, Fleming JE, Cadman DT, Blum $\mathrm{HM}$, et al. Outcome, prognosis, and risk in a longitudinal follow-up study. J Am Acad Child Adolesc Psychiatry 1992;31:916-23.

7. Clark LA, Watson D, Reynolds S. Diagnosis and classification of psychopathology: challenges to the current system and future directions. Annu Rev of Psychol 1995;46:121-52.

8. Morgado AF, Iguchi T, Bueno JR. Epidemiologia da dependência de drogas em grupos populacionais do Brasil. J Bras Psiquiatria 1983;32(5):281-92.

9. Carlini-Cotrim B, Carlini, EA. O consumo de solventes e outras drogas em crianças e adolescentes de baixa renda na Grande São Paulo. Parte I: estudantes de primeiro e segundo graus da rede estadual. Rev ABP-APAL 1987;8(2):49-58.

10. Carlini-Cotrim B, Carlini EA. O consumo de solventes e outras drogas em crianças e adolescentes de baixa renda na Grande São Paulo. Parte II: meninos de rua e menores internados. Rev ABP-APAL 1987;8(2):69-77.

11. Carlini EA, Nappo SA, Galduróz JC. A cocaína no Brasil ao longo dos últimos anos. Rev ABP-APAL 1993;15:121-7.

12. Noto AR, Galduróz JC, Carlini EA. Maconha: uma avaliação da situação brasileira através de quatro parâmetros epidemiológicos. Rev ABP-APAL 1995;17(4):133-7.

13. Scivoletto S, Henriques Jr. SG, Andrade AG. Uso de drogas por adolescentes que buscam atendimento ambulatorial: comparação entre "crack" e outras drogas ilícitas: um estudo piloto. Rev ABP-APAL 1997);19(1):7-17.

14. Galduróz JCF, Noto AR, Carlini EA. Têndencias do uso de drogas no Brasil: síntese dos resultados obtidos sobre o uso de drogas entre estudantes de $1^{\circ} \mathrm{e} 2^{\circ}$ graus em 10 capitais brasileiras. São Paulo: Centro Brasileiro de Informações Sobre Drogas Psicotrópicas - CEBRID/ UNIFESP; 1997.
15. Caldeira ZF, Lima ES, Dias PTP. Da teoria à prática. In: Caldeira ZF, org. Uma proposta de trabalho preventivo: da teoria à prática. Rio de Janeiro: Kat's Gráfica e Ed. NEPAD-UERJ/CN-DST/AIDS/MS/ UNDCP; 1998. p. 20-7.

16. Addiction Research Foundation Group. Youth \& drugs: an educational package for professionals. Workbook Unit 1: Adolescent development. Toronto: Addiction Research Foundation of Ontario; 1991.

17. Cruz MS. Abstinência de cocaína: um estudo de características psicopatológicas em dependentes que procuram tratamento. [dissertação]. Rio de Janeiro: Universidade Federal do Rio de Janeiro, 1996.

18. Valenzuela CF, Harris RA. Alcohol: neurobiology. In: Lowinson JH, Ruiz P, Millman RB, Langrod JG. Substance abuse. A comprehensive textbook. $3^{\text {rd }}$ ed. Baltimore: Williams \& Willkins; 1997. p. 119-42.

19. Gold MS. Cocaine (and crack): clinical aspects. In: Lowinson JH, Ruiz P, Millman RB, Langrod JG. Substance Abuse. A comprehensive textbook. $3^{\text {rd }}$ ed. Baltimore: Williams \& Willkins ; 1997. p. 181-99.

20. Ferri C. Cocaína: padrão de consumo e fatores associados à procura de tratamento. [tese]. São Paulo: Departamento de Psiquiatria da Universidade Federal de São Paulo, 1999.

21. Bukstein OG, Brent D, Kaminer Y. Comorbidity of substance abuse and other psychiatric disorders in adolescents. Am J Psychiatry 1989;146:1131-41.

22. Kaminer Y, Tarter RE, Bukstein OG, Kabene M. Staff, treatment completers, and noncompleters perceptions of the value of treatment variables. Am J Addict 1992;1:115-20.

23. Boyle MH, Offord D, Racine YA, Szatmari P, Fleming JE, Sanford MN. Identifying thresholds for classifying psychiatric disorder: issues and prospects. J Am Acad Child Adolesc Psychiatry 1996;35:1440-8.

24. Kazdin AE. Identifying depression in children: a comparison of alternative selection criteria. J Abnorm Child Psychol 1989;17:437-55.

25. Offord D, Boyle MH, Racine YA, Szatmari P, Fleming JE, Sanford $\mathrm{MN}$, et al. Integrating assessment data from multiple informants. J Am Acad Child Adolesc Psychiatry 1996;35:1078-85.

26. Winters KC, Henley GA. Assessing adolescents who abuse chemicals: the chemical dependency adolescent assessment project. In: Rahdert E, Grabowski J, eds. Adolescent drug abuse: analyses of treatment research. Washington (DC): DHHS Pub. (ADM) 88-1523. Supt. of Docs., U.S. Govt. Print. Off.; 1988. p. 4-8.

27. Winters KC, Stinchfield RD, Henly GA. Further validation of new scales measuring adolescent alcohol and other drug abuse. J Stud Alcohol 1992;54(5):534-41.

28. Weisz JR, Weiss B, Han SS, Granger DA, Morton T. Effects of psychotherapy with children and adolescents revisited: a meta-analysis of treatment outcome studies. Psychological Bull 1995;117:450-68.

29. Newcomb MD, Bentler PM. Substance use and abuse among children and teenagers. Am Psychol 1989;44:242-8. 
30. Wheeler K, Malmquist J. Treatment approaches in adolescents chemical dependency. Pediatr Clin North Am 1987;34(2):437-47.

31. Addiction Research Foundation Group Youth \& drugs: an educational package for professionals. Workbook Unit 5: Adolescent development. Toronto: Addiction Research Foundation of Ontario; 1991.

32. Bukstein OG, Van Hasselt VB. Alcohol and drug abuse. In: Bellack AS, Hersen M, eds. Handbook of behavior therapy in the psychiatric setting. New York: Plenum Press; 1993. p. 453-75.

33. Kazdin AE. The meanings and measurement of clinical significance. J Consul Clin Psychol 1999;67:332-9.

34. Cruz MS. Práticas médicas, toxicomanias e a promoção do exercício da cidadania. In: Acselrad G, org. Avessos do prazer: drogas, AIDS e direitos humanos. Rio de Janeiro: Ed. FIOCRUZ 2000. p. 233-43.

35. Bukstein OG, Kaminer Y. Nosology of adolescent substance abuse. Am J Addict 1994;3(1):1-13.

36. Addiction Research Foundation Group Youth \& drugs: an educational package for professionals. Workbook Unit 5: Adolescent development. Toronto: Addiction Research Foundation of Ontario; 1991.

37. Bandura A. Self-efficacy: towards a unifying theory of behavioral change. Psychol Rev 1977;84:199-215.
38. Hubbard RL, Cavanaugh ER, Craddock SG, Rachel JV. Characteristics, behaviors and outcomes for youth in TOPS. In: Friedman AS, Beschner GM, eds. Treatment services for adolescent substance abusers. Washington (DC): DHHS Pub. (ADM) 85-1342. Sup. of Docs., U.S. Govt. Print. Off.; 1985. p. 49-65.

39. Enrick CD. A review of psychologically oriented treatment of alcoholism. The relative effectiveness of different treatment approaches and the effectiveness of treatment versus no treatment. J Stud Alcohol 1975;36:88-108.

39. Catalano RF, Hawkins JD, Wells EA, Miller J, Brewer D. Evaluation of the effectiveness of adolescent drug abuse treatment, assessment of risks for relapse, and promising approaches for relapse prevention. Int J Addict 1990/1991;25(9A, 10A):1085-140.

40. Fleisch B. Approaches in the treatment of adolescents with emotional and substance abuse problems. Washington (DC): DHHS Pub. (ADM) 91-1744. Supt. of Docs., U.S. Govt. Print. Off; 1991.

41. National Institute on Drug Abuse (NIDA). Adolescent drug abuse: clinical assessment and therapeutic interventions. Research Monograph Series, 156. Washington (DC): U.S. Departament of Health and Human Services. Public Health Service. National Institutes of Health; 1995.

Correspondência: Ana Cecília Petta Roselli Marques

Rua Napoleão de Barros, 925 V. Clementino - 04024-002 São Paulo, SP 